\title{
Maternal sleep duration and neonatal birth weight: The Japan Environment and Children's Study
}

\author{
Tsuyoshi Murata ${ }^{1}$, Hyo Kyozuka ${ }^{1}$, Toma Fukuda ${ }^{1}$, Shun Yasuda ${ }^{1}$, Akiko Yamaguchi ${ }^{1}$, \\ Seiichi Morokuma ${ }^{2}$, Akiko Sato ${ }^{1}$, Yuka Ogata ${ }^{1}$, Kosei Shinoki ${ }^{1}$, Mitsuaki Hosoya ${ }^{1}$, Seiji \\ Yasumura $^{1}$, Koichi Hashimoto ${ }^{1}$, Hidekazu Nishigori ${ }^{1}$, and Keiya Fujimori ${ }^{1}$ \\ ${ }^{1}$ Fukushima Medical University \\ ${ }^{2}$ Graduate School of Medical Sciences, Kyushu University
}

August 17, 2020

\begin{abstract}
Objective: To evaluate the effect of maternal sleep duration (MSD) on low birth weight infants (LBW), small for gestational age infants (SGA), and macrosomia. Design: Prospective cohort study Setting: The Japan Environment and Children's Study (JECS) Population: Participants enrolled in JECS, with singleton pregnancies after 22 weeks, who gave birth between 2011 and 2014. Methods: Participants were categorized into five groups according to MSD during pregnancy: G1 (MSD <6.0 h), G2 $(6.0-7.9 \mathrm{~h})$, G3 $(8.0-8.9 \mathrm{~h}), \mathrm{G} 4(9.0-9.9 \mathrm{~h})$, and G5 (10.0-12.0 h). Main outcome measures: The effect of MSD on the risk of LBW $(<2,500 \mathrm{~g}$ and $<1,500 \mathrm{~g})$, SGA, and macrosomia $(>4,000 \mathrm{~g})$ with $\mathrm{G} 2$ as the reference, while adjusting for gestational excessive body weight gain (BWG). Analysis was also performed after stratification by gestational BWG. Results: We analyzed 82,171 participants. The adjusted odds ratios (aORs) of LBW <2,500 g in G4 and G5 and of SGA in G4 were 0.90 (95\% confidence interval [CI], 0.83-0.99), 0.86 (95\% CI, 0.76-0.99), and 0.91 (95\% CI, 0.82-0.99), respectively, before adjusting for excessive gestational BWG. No significant association was observed between MSD and these outcomes after adjusting for excessive gestational BWG. Among women with appropriate gestational BWG, the aORs of LBW $<2,500$ g and SGA in G4 were 0.88 (95\% CI, 0.80-0.97) and 0.87 (95\% CI, 0.78-0.97), respectively. Conclusion: This study revealed that 9.0-9.9 h of MSD significantly decreased LBW $<2,500 \mathrm{~g}$ and SGA in pregnant women with appropriate gestational BWG, relative to 6.0-7.9 $\mathrm{h}$ of MSD.
\end{abstract}

\section{Introduction}

Neonatal birth weight, which is related to perinatal morbidity and mortality, ${ }^{1-4}$ is affected by several obstetric complications, including preterm births (PTB), fetal growth restriction (FGR), and preeclampsia. ${ }^{5,6}$ Specifically, birth weight $<1,500 \mathrm{~g}$, defined as very low birth weight, is affected by severe prematurity and leads to increased mortality. ${ }^{7}$ Additionally, small for gestational age (SGA) infants have also been associated with neonatal and post-neonatal mortality. ${ }^{4,8,9}$ Further, LBW and SGA are associated with increased risk of coronary artery disease, diabetes mellitus, and arterial hypertension in adult life, as described in the Baker hypothesis ${ }^{10}$ which has been revised to the concept of developmental origins of health and disease (DOHaD) ${ }^{11}$ Conversely, macrosomia, defined as birth weight $>4,000 \mathrm{~g}$, is also associated with a risk of morbidity in infants. ${ }^{2,12}$

Several modifiable factors, including maternal pre-pregnancy body weight, gestational maternal body weight gain (BWG), and diet, have a major impact on the neonatal birth weight. ${ }^{5,12-14}$ Similarly, maternal sleep duration (MSD) during pregnancy also affects obstetric outcomes. ${ }^{15-17}$ However, the association between MSD and neonatal birth weight remains unclear. ${ }^{15,18}$ Previous studies have reported reduced MSD as a risk factor for SGA, ${ }^{19,20}$ but Morokuma et al. reported no association between MSD and SGA in a study including 
8,631 participants. ${ }^{21}$ Moreover, the appropriate MSD required to prevent LBW, SGA, and macrosomia has not been elucidated. As sleep is often disturbed in pregnant women, ${ }^{15}$ appropriate MSD would be a great concern for pregnant women.

The present study evaluated the effect of MSD on neonatal birth weight using the data from a nationwide Japanese prospective birth cohort study.

\section{Methods}

\section{Study design}

In this study, we used the data from the Japan Environment and Children's Study (JECS), which is a nationwide, government-funded, prospective birth cohort study that was started in January 2011 to investigate the effects of environmental factors on children's health. ${ }^{22,23}$ Briefly, JECS is funded directly by Japan's Ministry of the Environment and involves collaboration between the Programme Office (National Institute for Environmental Studies), the Medical Support Centre (National Centre for Child Health and Development), and 15 Regional Centres (Hokkaido, Miyagi, Fukushima, Chiba, Kanagawa, Koshin, Toyama, Aichi, Kyoto, Osaka, Hyogo, Tottori, Kochi, Fukuoka, and South Kyushu / Okinawa). ${ }^{23}$ The eligibility criteria for expectant mothers to participate in JECS were as follows: (1) residing in the study areas at the time of recruitment and expected to continually reside in Japan for the foreseeable future; (2) an expected delivery date between August 01, 2011 and mid-2014; and (3) capable of participating in the study without difficulty (i.e., able to comprehend the Japanese language and complete the self-administered questionnaires).

Either or both of the following recruitment protocols were applied: (1) recruitment at the time of the first prenatal examination at the cooperating obstetric facilities; and (2) recruitment at local government offices issuing a pregnancy journal, called the Maternal and Child Health Handbook, that is given to all expecting mothers in Japan before they receive municipal services for pregnancy, delivery, and childcare. We contacted pregnant women through cooperating health care providers and/or local government offices issuing Maternal and Child Health Handbooks and registered those willing to participate. Self-administered questionnaires, which were completed by the women during the first trimester and second/third trimester, were used to collect information on demographic factors, medical and obstetric history, physical and mental health, lifestyle, occupation, environmental exposure at home and in the workplace, housing conditions, and socioeconomic status. ${ }^{23}$

The JECS protocol was reviewed and approved by the Ministry of the Environment Institutional Review Board on Epidemiological Studies on March 23, 2010 (No. 15000141) ${ }^{22,23}$ and by the Ethics Committees of all participating institutions. The JECS was conducted in accordance with the principles of the Declaration of Helsinki and other national regulations and guidelines. Written informed consent was obtained from all participating women.

\section{Data collection}

The current analysis used the data set released in June 2016 (data set: jecs-ag-20160424). Specifically, we used three types of data: (1) M-T1, obtained from a self-reported questionnaire that was collected during the first trimester (the first questionnaire) and included questions regarding maternal medical background; (2) M-T2, obtained from a self-reported questionnaire that was collected during the second or third trimester (second questionnaire) and included questions regarding lifestyle and socioeconomic status; and (3) Dr-0m, collected from the medical records' transcripts provided by each participant's institution and that included

obstetrical outcomes such as gestational age, birth weight, neonatal sex, and maternal body weight. The participants with singleton pregnancies after 22 weeks were included in the present study. The women with missing information were excluded from the analysis.

\section{Exposure variables}

MSD was calculated using the data of questionnaire in M-T2. ${ }^{24}$ The participants were categorized into five groups according to MSD during pregnancy: G1 (MSD < 6.0 h), G2 (MSD 6.0-7.9 h), G3 (MSD 8.0-8.9 
h), G4 (MSD 9.0-9.9 h), and G5 (MSD 10.0-12.0 h) based on the report of Ministry of Health, Labour and Welfare, Japan, ${ }^{25}$ which suggests $6.0-7.9 \mathrm{~h}$ of sleep as the appropriate sleep duration for general adults to prevent several health problems. The participants who reported MSD $>12.0 \mathrm{~h}$ were excluded from the present study because these participants were considered to have reported inaccurate MSD.

\section{Obstetric outcomes and confounding factors}

LBW was classified into two categories: LBW $<2,500 \mathrm{~g}$ and LBW $<1,500$ g. ${ }^{1}$ SGA was defined as a birth weight below 1.5 standard deviations, corrected for gestational age and sex, according to "New Japanese neonatal anthropometric charts for gestational age at birth". ${ }^{8}$ Macrosomia was defined as neonatal birth weight $>4,000$ g. ${ }^{12}$

The following parameters were considered as potential confounding factors: maternal age, body mass index (BMI) before pregnancy, parity, maternal smoking status, maternal alcohol consumption status, maternal educational status, annual household income, PTB before 37 weeks, and gestational BWG. Maternal age was categorized into three age groups: <20 years, 20-29 years, and [?]30 years based on a previous study that showed that maternal age was related to certain obstetric outcomes, such as PTB, LBW, and SGA. ${ }^{26}$ BMI before pregnancy was categorized into three groups $\left(<18.5 \mathrm{~kg} / \mathrm{m}^{2}, 18.5-25.0 \mathrm{~kg} / \mathrm{m}^{2}\right.$, and $>25.0$ $\left.\mathrm{kg} / \mathrm{m}^{2}\right) \cdot{ }^{27,28}$ Parity was categorized into two groups (nulliparous and multiparous). Maternal participants were requested to provide information about their smoking status by choosing one of the following: "Yes, I still smoke"; "Never"; "Previously did but quit before recognizing current pregnancy"; and "Previously did but quit after learning of current pregnancy." The maternal participants who chose "Yes, I still smoke" comprised the smoking category, whereas the other participants comprised the non-smoking category. Maternal participants were requested to provide information about their alcohol consumption status by choosing one of the following: "never drank," "quit drinking before pregnancy," "quit drinking during early pregnancy," and "kept drinking during pregnancy." ${ }^{29}$ The maternal participants who chose "kept drinking during pregnancy" comprised the drinking category, while the other participants comprised the non-drinking category. The educational status of the mother was categorized into four groups on the basis of the number of years of education (junior high school, <10 years; high school, 10-12 years; professional school or university, 1316 years; and graduate school, [?]17 years). Annual household income was categorized into four groups (<2,000,000 JPY, 2,000,000-5,999,999 JPY, 6,000,000-9,999,999 JPY, and [?]10,000,000 JPY). PTB was defined as the birth before 37 weeks of gestation, the record of which was collected from Dr-0m data. The data for gestational BWG were obtained from Dr-0m data, which included information about body weight before pregnancy $(\mathrm{kg})$ and body weight just before delivery $(\mathrm{kg})$. Gestational BWG was defined as body weight just before delivery minus body weight before pregnancy $(\mathrm{kg})$. We defined appropriate BWG as less than $12 \mathrm{~kg}$ and excessive BWG as more than $12 \mathrm{~kg}$, according to the criteria aimed at appropriate birth weight, described by Ministry of Health, Labour and Welfare, Japan. ${ }^{27,28}$ These confounding factors were chosen on the basis of the clinical importance. ${ }^{5,13,14}$

\section{Statistical analyses}

Participant characteristics were summarized according to the maternal sleeping status. All variables were found to follow a normal distribution based on the Shapiro-Wilk test. One-way analysis of variance was used to compare continuous variables among different MSD groups, and the chi-square test was used to compare categorical variables.

Initially, adjusted odds ratios (aORs) and 95\% confidence intervals (CIs) for LBW $<2,500 \mathrm{~g}$, LBW $<1,500 \mathrm{~g}$, SGA, and macrosomia were calculated using a multiple logistic regression model, with women in G2 as the reference. In Model 1, aOR for LBW was adjusted for maternal age, BMI before pregnancy, parity, maternal smoking status, maternal alcohol consumption status, maternal educational status, annual household income, and PTB before 37 weeks. aOR for SGA was adjusted for maternal age, BMI before pregnancy, maternal smoking status, maternal alcohol consumption status, maternal educational status, and annual household income. aOR for macrosomia was adjusted for maternal age, BMI before pregnancy, parity, maternal smoking status, maternal alcohol consumption status, maternal educational status, and annual household income. In 
Model 2, excessive gestational BWG was added as a confounding factor (in addition to those of Model 1) to calculate aORs for these outcomes.

Further, we stratified the participants on the basis of gestational BWG, and aORs for LBW $<2,500 \mathrm{~g}$ and SGA were calculated using a multiple logistic regression model, with women in G2 as the reference, using the comparable confounding factors in Model 1.

SPSS version 26 (IBM Corp., Armonk, NY, USA) was used for statistical analyses. Differences with p-values $<0.05$ were considered statistically significant.

\section{Results}

The total number of fetal records during 2011-2014 was 104,102. After applying our inclusion criteria, 82,171 participants were eligible for this study (Figure 1). Among 82,171 participants, 3,958 (4.8\%) were in G1, $37,944(46.2 \%)$ in G2, 23,769 (28.9\%) in G3, 11,976 (14.6\%) in G4, and 4,524 (5.5\%) in G5.

Table 1 summarizes the maternal background and obstetric outcomes based on the MSD groups. All maternal background characteristics were significantly affected by MSD. Birth weight increased significantly, and the incidence of LBW $<2,500 \mathrm{~g}$ was significantly lower in G4 and G5. No significant differences were observed in the incidence of PTB, LBW $<1,500 \mathrm{~g}$, SGA, and macrosomia among the groups.

Table 2 shows the aORs of LBW $<2,500 \mathrm{~g}$, LBW $<1,500 \mathrm{~g}$, SGA, and macrosomia among all groups with G2 as a reference. No significant association was observed between MSD and these outcomes after adjusting for excessive gestational BWG in model 2. Additionally, there was no significant association between MSD and $\mathrm{LBW}<1,500 \mathrm{~g}$ and macrosomia.

Table 3 shows the aORs of LBW $<2,500 \mathrm{~g}$ and SGA among all groups with G2 as a reference, after stratification by gestational BWG. No significant association was observed between MSD and these outcomes in women with excessive gestational BWG.

\section{Discussion}

\section{Main findings}

The present study suggests that $>9 \mathrm{~h}$ of MSD decreased the incidence of LBW $<2,500 \mathrm{~g}$ and SGA but did not affect the outcome after adjusting for excessive gestational BWG, which implies that both long MSD and excessive gestational BWG had great impact on decreasing the risk of LBW and SGA. Additionally, 9 to $<10 \mathrm{~h}$ of MSD significantly decreased the risk of LBW $<2,500 \mathrm{~g}$ and SGA in women with appropriate gestational BWG.

\section{Interpretation}

The present study, using a large prospective cohort, showed that both MSD and gestational BWG affected neonatal birth weight. Previous studies with relatively small sample sizes have shown a conflicting relationship between MSD and neonatal birth weight. ${ }^{15,19-21}$ On the basis of the findings of the present study, we speculate that long MSD, along with gestational BWG, decreases the incidence of LBW and SGA. ${ }^{14}$ Although the effect of MSD on gestational BWG is not clearly defined, ${ }^{30,31}$ recent studies have reported that excessive sleep duration increased obesity in non-pregnant adults. ${ }^{32,33}$ The fetuses in mothers with excessive gestational BWG may receive more nutrients and fetal growth through increased plasma volume, which may increase cardiac output and utero-placental blood flow compared to those in mothers with insufficient gestational BWG. ${ }^{34,35}$ Therefore, sufficient BWG by proper diet and sufficient MSD is required to prevent LBW and SGA; however, the concerns about disadvantages of maternal obesity prevail. ${ }^{36,37}$

Conversely, the direct effect of MSD on obstetric outcomes in the appropriate gestational BWG group has not been yet reported. As well as maternal inflammatory stress has been reported to be related to several obstetric outcomes such as PTB, FGR, and preeclampsia, ${ }^{38-40}$ previous studies have also reported that disturbed maternal sleep may cause adverse obstetric outcomes, with augmentation of maternal inflammatory response. ${ }^{15,41}$ Increased inflammation may interfere with the remodeling of spiral arteries in the placenta, 
thereby leading to PTB, FGR, and preeclampsia. ${ }^{42-44}$ Thus, preventing reduced MSD may reduce maternal inflammation and prevent these adverse obstetric outcomes. Further, maternal inflammatory stress is also affected by lifestyle, including dietary habits and exercises ${ }^{45,46}$; and comprehensive modification of these lifestyles may help in reducing inflammatory stress.

However, $>10 \mathrm{~h}$ of MSD did not decrease the incidence of LBW and SGA in women with appropriate gestational BWG, which implies that $>10 \mathrm{~h}$ of MSD did not affect neonatal birth weight. This may be because $>10 \mathrm{~h}$ of MSD might be affected by maternal diseases, conditions, and behaviors, such as depression, excessive mental stress, and use of sleeping pills, ${ }^{24,47,48}$ which may potentially decrease the neonatal birth weight. ${ }^{49-51}$ Moreover, because too long MSD may have other unfavorable effects including excessive BWG, ${ }^{32,33}$ we do not suggest that pregnant women should have $>10 \mathrm{~h}$ of sleep.

\section{Strengths and limitations}

The strength of the present study is that the aORs of LBW and SGA provide clear information for perinatal counseling. Owing to the large study population, including $>80,000$ participants, our results must be reliable. Since pregnant women have more sleep problems, affected by gestational age and hormonal changes, ${ }^{24}$ than their non-pregnant counterparts, the effect of MSD on fetal and neonatal health may be a great concern for pregnant women. There is no consensus on appropriate MSD required to prevent adverse obstetric outcomes. Therefore, the present study may help to suggest adequate MSD required to prevent LBW and SGA.

The present study has several limitations. First, MSD in the present study is based on self-reported questionnaire data, which might have resulted in an inaccurate calculation of actual MSD. In addition, MSD is a volatile index because it varies daily in the individuals and may vary with gestational age. ${ }^{24}$ Careful interpretation is needed regarding these instabilities of MSD. Further study with polysomnography and unified gestational age may preclude this limitation. Second, we did not account for the quality of sleep by evaluating factors such as time zone, division, where to sleep, and with whom to sleep. We evaluated MSD as a simple quantitative measurement of maternal sleep. Careful interpretation of the results is needed because the quality of maternal sleep, in addition to MSD, may also affect obstetric outcomes.

\section{Conclusion}

This study revealed that both $>9 \mathrm{~h}$ of MSD and excessive gestational BWG had great impact on the incidence of LBW and SGA, and 9 to $<10 \mathrm{~h}$ of MSD decreased the risk of LBW and SGA in women with appropriate gestational BWG. It is important for care providers to provide the latest data for proper counseling regarding the association between MSD and neonatal birth weight and suggest comprehensive modifications in lifestyles of pregnant women, including sufficient MSD, to prevent LBW and SGA. Furthermore, because JECS is a prospective cohort study, future elucidation of long-term childhood outcomes on the basis of MSD and neonatal birthweight would strengthen the conclusion of this study.

Acknowledgements: The authors are grateful to all the participants of the study and to the Ministry of the Environment, Japan. Members of the JECS Group as of 2020 are: Michihiro Kamijima (principal investigator, Nagoya City University, Nagoya, Japan), Shin Yamazaki (National Institute for Environmental Studies, Tsukuba, Japan), Yukihiro Ohya (National Center for Child Health and Development, Tokyo, Japan), Reiko Kishi (Hokkaido University, Sapporo, Japan), Nobuo Yaegashi (Tohoku University, Sendai, Japan), Koichi Hashimoto (Fukushima Medical University, Fukushima, Japan), Chisato Mori (Chiba University, Chiba, Japan), Shuichi Ito (Yokohama City University, Yokohama, Japan), Zentaro Yamagata (University of Yamanashi, Chuo, Japan), Hidekuni Inadera (University of Toyama, Toyama, Japan), Takeo Nakayama (Kyoto University, Kyoto, Japan), Hiroyasu Iso (Osaka University, Suita, Japan), Masayuki Shima (Hyogo College of Medicine, Nishinomiya, Japan), Youichi Kurozawa (Tottori University, Yonago, Japan), Narufumi Suganuma (Kochi University, Nankoku, Japan), Koichi Kusuhara (University of Occupational and Environmental Health, Kitakyushu, Japan), and Takahiko Katoh (Kumamoto University, Kumamoto, Japan).

Disclosure of interests: The authors report no conflicts of interest.

Contribution to Authorship: T.M. conceptualized and designed the study. T.M., H.K., T.F., S.Y., A.Y., 
S.M., K.H., H.N., and K.F. contributed to the study design. K.S., A.S., and Y.O. collected the data. T.M. analyzed the data and wrote the manuscript. M.H., S.Y., K.H., K.S., A.S., Y.O., H.N., K.F., and the JECS group reviewed the manuscript and provided critical advice. All authors approved the final manuscript.

Details of Ethical Approval: The JECS protocol was reviewed and approved by the Ministry of the Environment Institutional Review Board on Epidemiological Studies on March 23, 2010 (No. 15000141) ${ }^{22,23}$ and by the Ethics Committees of all participating institutions. The JECS was conducted in accordance with the principles of the Declaration of Helsinki and other national regulations and guidelines. Written informed consent was obtained from all participating women.

Funding: The Japan Environment and Children's Study was funded by the Ministry of the Environment, Japan. The findings and conclusions of this article are solely the responsibility of the authors and do not represent the official views of the Ministry of the Environment, Japan.

\section{References}

[1] Hughes MM, Black RE, Katz J. 2500-g Low birth weight cutoff: History and implications for future research and policy. Matern Child Health J. 2017;21:283-9.

[2] Wilcox AJ. On the importance-and the unimportance-of birthweight. Int J Epidemiol. 2001;30:1233-41.

[3] Lawn JE, Cousens S, Zupan J, Lancet Neonatal Survival Steering Team. 4 million neonatal deaths: When? Where? Why? Lancet. 2005;365:891-900.

[4] Katz J, Lee AC, Kozuki N, Lawn JE, Cousens S, Blencowe H, et al. Mortality risk in preterm and smallfor-gestational-age infants in low-income and middle-income countries: A pooled country analysis. Lancet. 2013;382:417-25.

[5] Kramer MS. Determinants of low birth weight: Methodological assessment and meta-analysis. Bull World Health Organ. 1987;65:663-737.

[6] Nafday SM. Abnormalities of fetal growth. In: Campbell DE, editor. Neonatology for primary care. American Academy of Pediatrics; 2015. p. 323-44.

[7] McLeod JS, Menon A, Matusko N, Weiner GM, Gadepalli SK, Barks J, et al. Comparing mortality risk models in VLBW and preterm infants: Systematic review and meta-analysis. J Perinatol. 2020;40:695-703.

[8] Itabashi K, Miura F, Uehara R, Nakamura Y. New Japanese neonatal anthropometric charts for gestational age at birth. Pediatr Int. 2014;56:702-8.

[9] Pulver LS, Guest-Warnick G, Stoddard GJ, Byington CL, Young PC. Weight for gestational age affects the mortality of late preterm infants. Pediatrics. 2009;123:e1072-7.

[10] Barker DJ, Osmond C. Infant mortality, childhood nutrition, and ischaemic heart disease in England and Wales. Lancet. 1986;1:1077-81.

[11] Gluckman PD, Hanson MA. Living with the past: Evolution, development, and patterns of disease. Science. 2004;305:1733-6.

[12] Committee on Practice Bulletins-Obstetrics. Macrosomia: ACOG Practice Bulletin, Number 216. Obstet Gynecol. 2020;135:e18-e35.

[13] Kramer MS. The epidemiology of adverse pregnancy outcomes: An overview. J Nutr. 2003;133:1592S-6S.

[14] Goldstein RF, Abell SK, Ranasinha S, Misso M, Boyle JA, Black MH, et al. Association of gestational weight gain with maternal and infant outcomes: A systematic review and meta-analysis. JAMA. 2017;317:2207-25.

[15] Okun ML, Roberts JM, Marsland AL, Hall M. How disturbed sleep may be a risk factor for adverse pregnancy outcomes. Obstet Gynecol Surv. 2009;64:273-80. 
[16] Heazell A, Li M, Budd J, Thompson J, Stacey T, Cronin RS, et al. Association between maternal sleep practices and late stillbirth - findings from a stillbirth case-control study. BJOG. 2018;125:254-62.

[17] Myoga M, Tsuji M, Tanaka R, Shibata E, Askew DJ, Aiko Y, et al. Impact of sleep duration during pregnancy on the risk of gestational diabetes in the Japan environmental and Children's study (JECS). BMC Pregnancy Childbirth. 2019;19:483.

[18] Warland J, Dorrian J, Morrison JL, O'Brien LM. Maternal sleep during pregnancy and poor fetal outcomes: A scoping review of the literature with meta-analysis. Sleep Med Rev. 2018;41:197-219.

[19] Abeysena C, Jayawardana P, DE A Seneviratne R. Maternal sleep deprivation is a risk factor for small for gestational age: a cohort study. Aust N Z J Obstet Gynaecol. 2009;49:382-7.

[20] Franco-Sena AB, Kahn LG, Farias DR, Ferreira AA, Eshriqui I, Figueiredo ACC, et al. Sleep duration of $24 \mathrm{~h}$ is associated with birth weight in nulli- but not multiparous women. Nutrition. 2018;55-56:91-8.

[21] Morokuma S, Shimokawa M, Kato K, Sanefuji M, Shibata E, Tsuji M, et al. Maternal sleep and small for gestational age infants in the Japan Environment and Children's Study: A cohort study. BMC Res Notes. 2017;10:394.

[22] Kawamoto T, Nitta H, Murata K, Toda E, Tsukamoto N, Hasegawa M, et al. Rationale and study design of the Japan Environment and Children's Study (JECS). BMC Public Health. 2014;14:25.

[23] Michikawa T, Nitta H, Nakayama SF, Yamazaki S, Isobe T, Tamura K, et al. Baseline profile of participants in the Japan Environment and Children's Study (JECS). J Epidemiol. 2018;28:99-104.

[24] Konishi M, Tomotaki A, Yamamoto-Hanada K, Mezawa H, Ayabe T, Ishitsuka K, et al. Sleep status varies by age among Japanese women during preconception and pregnancy in a nationwide birth cohort study [the Japan Environment and Children's Study (JECS)]. Sleep Biol Rhythms. 2018;17:161-72.

[25] Ministry of Health, Labour and Welfare, Japan. Sleep guidelines for health promotion (in Japanese). Available at:https://www.mhlw.go.jp/file/06-Seisakujouhou-10900000-Kenkoukyoku/0000047221.pdf. Accessed on August 15, 2020.

[26] Kyozuka H, Fujimori K, Hosoya M, Yasumura S, Yokoyama T, Sato A, et al. The effect of maternal age at the first childbirth on gestational age and birth weight: The Japan Environment and Children's Study (JECS). J Epidemiol. 2019;29:187-91.

[27] Minakami H, Maeda T, Fujii T, Hamada H, Iitsuka Y, Itakura A, et al. Guidelines for obstetrical practice in Japan: Japan Society of Obstetrics and Gynecology (JSOG) and Japan Association of Obstetricians and Gynecologists (JAOG) 2014 edition. J Obstet Gynaecol Res. 2014;40:1469-99.

[28] Ministry of Health, Labour and Welfare, Japan. Guidelines for health promotion in pregnant women (in Japanese). Available at:https://www.mhlw.go.jp/houdou/2006/02/h0201-3a.html. Accessed on August $15,2020$.

[29] Yokoyama Y, Takachi R, Ishihara J, Ishii Y, Sasazuki S, Sawada N, et al. Validity of short and long selfadministered food frequency questionnaires in ranking dietary intake in middle-aged and elderly Japanese in the Japan Public Health Center-based Prospective Study for the Next Generation (JPHC-NEXT) protocol area. J Epidemiol. 2016;26:420-32.

[30] Gay CL, Richoux SE, Beebe KR, Lee KA. Sleep disruption and duration in late pregnancy is associated with excess gestational weight gain among overweight and obese women. Birth. 2017;44:173-80.

[31] Balieiro LCT, Gontijo CA, Fahmy WM, Maia YCP, Crispim CA. Does sleep influence weight gain during pregnancy? A prospective study. Sleep Sci. 2019;12:156-64.

[32] Jike M, Itani O, Watanabe N, Buysse DJ, Kaneita Y. Long sleep duration and health outcomes: A systematic review, meta-analysis and meta-regression. Sleep Med Rev. 2018;39:25-36. 
[33] Tan X, Chapman CD, Cedernaes J, Benedict C. Association between long sleep duration and increased risk of obesity and type 2 diabetes: A review of possible mechanisms. Sleep Med Rev. 2018;40:127-34.

[34] Neggers Y, Goldenberg RL. Some thoughts on body mass index, micronutrient intakes and pregnancy outcome. J Nutr. 2003;133:1737S-40S.

[35] Rosso P, Salas SP. Mechanism of fetal growth retardation in the underweight mother. In: Allen L, King J, Lonnerdal B, editors. Nutrient regulation during pregnancy, lactation, and infant growth. New York: Plenum Press; 1994. p. 1-9.

[36] Bogaerts A, Van den Bergh BR, Ameye L, Witters I, Martens E, Timmerman D, et al. Interpregnancy weight change and risk for adverse perinatal outcome. Obstet Gynecol. 2013;122:999-1009.

[37] Villamor E, Cnattingius S. Interpregnancy weight change and risk of adverse pregnancy outcomes: A population-based study. Lancet. 2006;368:1164-70.

[38] Romero R, Espinoza J, Gonçalves LF, Kusanovic JP, Friel LA, Nien JK. Inflammation in preterm and term labour and delivery. Semin Fetal Neonatal Med. 2006;11:317-26.

[39] Bartha JL, Romero-Carmona R, Comino-Delgado R. Inflammatory cytokines in intrauterine growth retardation. Acta Obstet Gynecol Scand. 2003;82:1099-102.

[40] Freeman DJ, McManus F, Brown EA, Cherry L, Norrie J, Ramsay JE, et al. Short- and long-term changes in plasma inflammatory markers associated with preeclampsia. Hypertension. 2004;44:708-14.

[41] Chang JJ, Pien GW, Duntley SP, Macones GA. Sleep deprivation during pregnancy and maternal and fetal outcomes: Is there a relationship? Sleep Med Rev. 2010;14:107-14.

[42] Fluhr H, Krenzer S, Stein GM, Stork B, Deperschmidt M, Wallwiener D, et al. Interferon-gamma and tumor necrosis factor-alpha sensitize primarily resistant human endometrial stromal cells to Fas-mediated apoptosis. J Cell Sci. 2007;120:4126-33.

[43] Salamonsen LA, Hannan NJ, Dimitriadis E. Cytokines and chemokines during human embryo implantation: Roles in implantation and early placentation. Semin Reprod Med. 2007;25:437-44.

[44] Arias F, Rodriquez L, Rayne SC, Kraus FT. Maternal placental vasculopathy and infection: Two distinct subgroups among patients with preterm labor and preterm ruptured membranes. Am J Obstet Gynecol. 1993;168:585-91.

[45] Ishibashi M, Kyozuka H, Yamaguchi A, Fujimori K, Hosoya M, Yasumura S, et al. Effect of proinflammatory diet before pregnancy on gestational age and birthweight: The Japan Environment and Children's Study. Matern Child Nutr. 2020;16:e12899.

[46] Stigger FS, Zago Marcolino MA, Portela KM, Plentz RDM. Effects of exercise on inflammatory, oxidative, and neurotrophic biomarkers on cognitively impaired individuals diagnosed with dementia or mild cognitive impairment: A systematic review and meta-analysis. J Gerontol A Biol Sci Med Sci. 2019;74:616-24.

[47] Lopez R, Barateau L, Evangelista E, Dauvilliers Y. Depression and hypersomnia: A complex association. Sleep Med Clin. 2017;12:395-405.

[48] Grigolon RB, Trevizol AP, Cerqueira RO, Lee Y, Mansur RB, McIntyre RS, et al. Hypersomnia and bipolar disorder: A systematic review and meta-analysis of proportion. J Affect Disord. 2019;246:659-66.

[49] Grote NK, Bridge JA, Gavin AR, Melville JL, Iyengar S, Katon WJ. A meta-analysis of depression during pregnancy and the risk of preterm birth, low birth weight, and intrauterine growth restriction. Arch Gen Psychiatry. 2010;67:1012-24.

[50] Li X, Gao R, Dai X, Liu H, Zhang J, Liu X, et al. The association between symptoms of depression during pregnancy and low birth weight: A prospective study. BMC Pregnancy Childbirth. 2020;20:147. 
[51] Wang LH, Lin HC, Lin CC, Chen YH, Lin HC. Increased risk of adverse pregnancy outcomes in women receiving zolpidem during pregnancy. Clin Pharmacol Ther. 2010;88:369-74.

\section{Table legends}

Table 1. Maternal medical background and obstetric outcomes of participants according to maternal sleep duration status

Table 2. Crude odds ratios (cORs), adjusted odds ratios (aORs), and 95\% confidence interval (CI) of obstetric complications stratified by maternal sleep duration

Table 3. Crude odds ratios (cORs), adjusted odds ratios (aORs), and 95\% confidence interval (CI) of low birth weight infants $<2,500 \mathrm{~g}$ and small for gestational age infants in women with appropriate and excessive gestational body weight gain

\section{Figure legends}

Figure 1. Flowchart depicting enrollment of the participants in the study

\section{Hosted file}

Table 1.docx available at https://authorea.com/users/327152/articles/476071-maternal-sleepduration-and-neonatal-birth-weight-the-japan-environment-and-children-s-study

\section{Hosted file}

Table 2.docx available at https://authorea.com/users/327152/articles/476071-maternal-sleepduration-and-neonatal-birth-weight-the-japan-environment-and-children-s-study

\section{Hosted file}

Table 3.docx available at https://authorea.com/users/327152/articles/476071-maternal-sleepduration-and-neonatal-birth-weight-the-japan-environment-and-children-s-study

\section{Hosted file}

Figure 1.docx available at https://authorea.com/users/327152/articles/476071-maternal-sleepduration-and-neonatal-birth-weight-the-japan-environment-and-children-s-study 